\title{
Calibration of a High Resolution Soft X-ray Spectrometer
}

J. Dunn, P. Beiersdorfer, G. V. Brown, E. W. Magee

February 5, 2010

11th International Conference on X-ray Lasers Belfast, United Kingdom

August 17, 2008 through August 22, 2008 
This document was prepared as an account of work sponsored by an agency of the United States government. Neither the United States government nor Lawrence Livermore National Security, LLC, nor any of their employees makes any warranty, expressed or implied, or assumes any legal liability or responsibility for the accuracy, completeness, or usefulness of any information, apparatus, product, or process disclosed, or represents that its use would not infringe privately owned rights. Reference herein to any specific commercial product, process, or service by trade name, trademark, manufacturer, or otherwise does not necessarily constitute or imply its endorsement, recommendation, or favoring by the United States government or Lawrence Livermore National Security, LLC. The views and opinions of authors expressed herein do not necessarily state or reflect those of the United States government or Lawrence Livermore National Security, LLC, and shall not be used for advertising or product endorsement purposes. 


\title{
Calibration of a High Resolution Soft X-ray Spectrometer
}

\author{
J. Dunn, P. Beiersdorfer, G.V. Brown and E.W. Magee \\ Lawrence Livermore National Laboratory, Physical Sciences Directorate, \\ Livermore, CA 94551, USA
}

\begin{abstract}
A high resolution grating spectrometer (HRGS) with 2400 line $/ \mathrm{mm}$ variable line spacing grating for the $10-50 \AA$ wavelength range has been designed for laser-produced plasma experiments at the Lawrence Livermore National Laboratory (LLNL). The spectrometer has a large radius of curvature, $\mathrm{R}=44.3 \mathrm{~m}$, is operated at a $2^{\circ}$ grazing angle and can record high signal-to-noise spectra when used with a low-noise, cooled, charge-coupled device detector. The instrument can be operated with a $10-25 \mu \mathrm{m}$ wide slit to achieve the best spectral resolving power on laser plasma sources, approaching 2000, or in slitless mode with a small symmetrical emission source. Results will be presented for the spectral response of the spectrometer cross-calibrated at the LLNL Electron Beam Ion Trap facility using the broadband x-ray energy EBIT Calorimeter Spectrometer (ECS).
\end{abstract}

\section{Introduction}

A high resolution grating spectrometer (HRGS) has been designed to make measurements of spectral line shapes as well as precise wavelength positions of highly ionized lines emitted from laser-produced plasmas [1]. For this study, the main lines of interest are the K-shell oxygen and carbon series lines in the $10-50 \AA$ wavelength band. An important part of this work is to be able to diagnose the emission conditions of these plasmas, in particular the electron temperature $T_{e}$ and density $n_{e}$. Several spectroscopic methods have been described that allow the determination of these plasma parameters [2, 3]. These include Stark broadening of the higher $\mathrm{n}$ transitions (for $n_{e}$ ), densitysensitive dielectronic satellite line intensity ratios, x-ray continuum slope (for $T_{e}$ ), resonance line intensity ratios in the He-like and H-like ion series (for $T_{e}$ ) as well as satellite to resonance line ratios (for $T_{e}$ ). Knowledge of the instrument spectral sensitivity response curve across the waveband is required to determine accurately some of the above electron temperature parameters. Previous work calibrating a $2400 \mathrm{line} / \mathrm{mm}$ flat field variable spacing grating spectrometer was achieved by observing a laser-produced plasma source simultaneously with a transmission grating spectrometer of known efficiency [4]. In this work we report the calibration of the HRGS instrument using the SuperEBIT electron beam ion trap x-ray source located at LLNL. The spectral sensitivity consisting of the grating reflectivity $R(E)$ and detector quantum 
efficiency $Q(E)$ are determined against the EBIT Calorimeter Spectrometer (ECS) [5]. The thin $200 \mathrm{~nm}$ aluminum foil filter used as a light tight filter in the laser plasma experiments is calibrated separately using the ECS and SuperEBIT [6].

In the next section the HRGS instrument, the SuperEBIT source, the ECS and the methods for calibrating the spectral sensitivity are described in detail. In section 3 the results of the measurements are presented and compared with other values in the literature.

\section{Experimental Description}

The high resolution grating spectrometer uses a large radius of curvature, $R=44.3 \mathrm{~m}, 2400$ line $/ \mathrm{mm}$ variable-spaced grating as the wavelength dispersion element. The grating, with a large active area of $10 \times 5 \mathrm{~cm}^{2}(L \times W)$, is inclined at an angle of incidence of approximately $2^{\circ}$ to the source. The instrument is run on laser-produced plasma experiments with a $25 \mu \mathrm{m}$ slit placed at $150 \mathrm{~cm}$ from the grating and defines the instrument spectral resolution. For the operation on SuperEBIT the slit was removed and the instrument was set up to focus onto the $\mathrm{x}$-ray emission region of the ion trap. A low noise LN-cooled, back-thinned CCD $1300 \times 1340\left(20 \times 20 \mu \mathrm{m}^{2}\right.$ pixel $)$ charge-coupled device records the spectrum and is located approximately 150 $\mathrm{cm}$ from the grating. This instrument is enclosed in a separate vacuum chamber and is bolted and aligned to the laser target or SuperEBIT x-ray source [1]. Single-shot spectra with lines resolved to about one part in 2000 are recorded from the laser-irradiated targets with energy on target of $0.5 \mathrm{~J}$ or more. For the SuperEBIT calibration runs the integration times were varied from 10 minutes to 60 minutes to record a spectrum with good line intensity. Figure 1 shows the layout of the HRGS instrument on SuperEBIT.

The LLNL EBIT-I was first built in 1986. SuperEBIT has been in operation for over 15 years [7]. It has been used to generate precise atomic data, detailed spectroscopy of highly charged ions as well as a calibration source for x-ray instrumentation. For this study neutral atoms using the gases $\mathrm{CO}_{2}, \mathrm{Kr}, \mathrm{Ne}$, and $\mathrm{SF}_{6}$, were injected into the trap and collisionally ionized by the electron beam. The beam electrons are confined and focused by a $3 \mathrm{~T}$ magnetic field generated by superconducting Helmoltz coils. The ions are longitudinally confined by three voltages applied to the drift tube. Radial confinement is produced by the electron beam. This produces a trap dimension of $2 \mathrm{~cm} \times 60$ $\mu \mathrm{m}(H \times W)$ where the electron density $\mathrm{n}_{\mathrm{e}}$ is in the range $2 \times 10^{10}-5 \times 10^{12}$ $\mathrm{cm}^{-3}$. The ionization of the gas is adjusted by the electron beam voltage and the trap time defined by gating the drift voltages.

A second instrument very similar to the HRGS designed for use on EBIT used the same 2400 line $/ \mathrm{mm} \mathrm{R}=44.3 \mathrm{~m}, 2400$ line $/ \mathrm{mm}$ grating and a LN- 
cooled CCD as described above [8]. It was set up like the HRGS and run in the same way where the instrument was focused on the EBIT x-ray source.

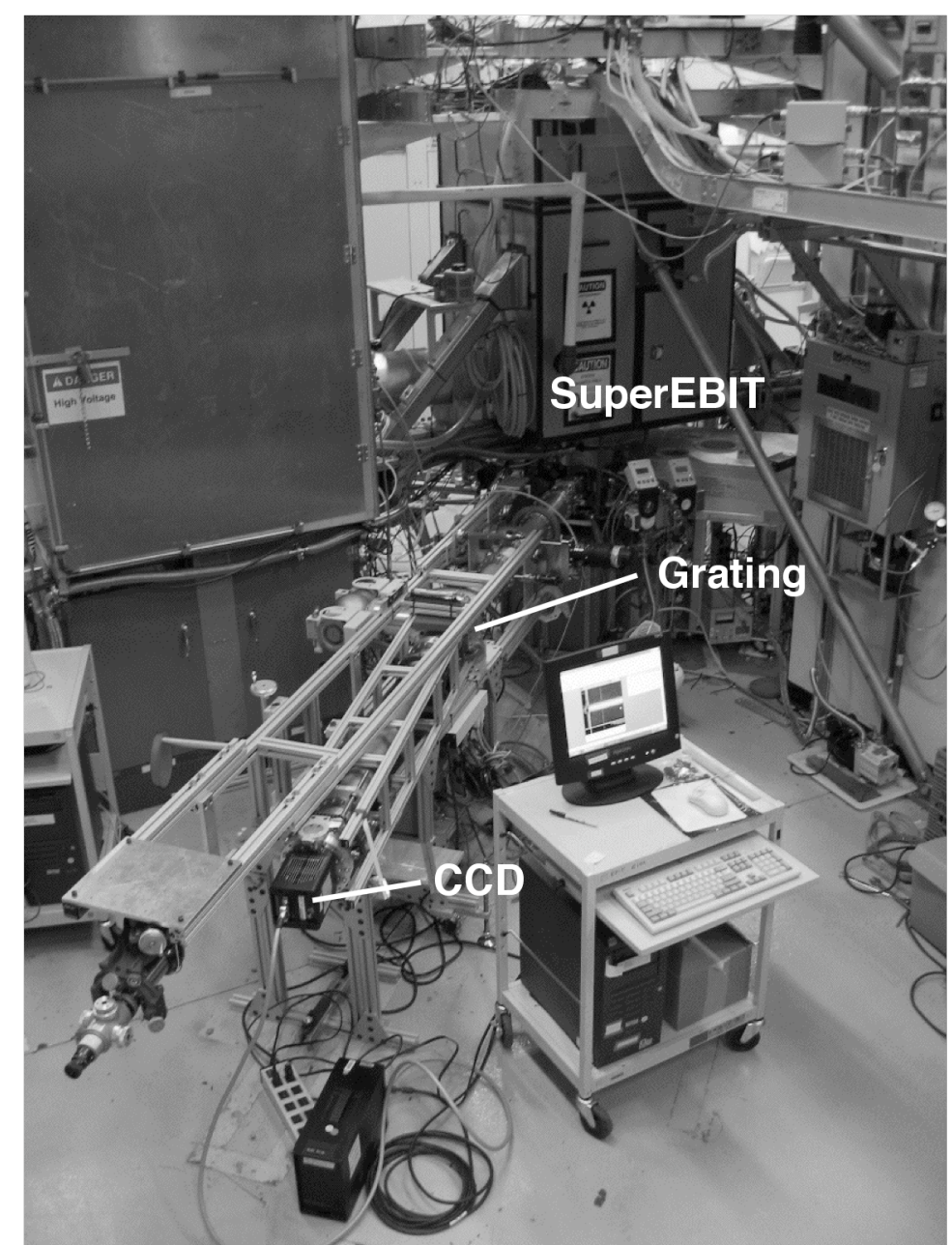

Fig. 1. High resolution grating spectrometer on the LLNL SuperEBIT. Grating and $\mathrm{CCD}$ detector are separated by $150 \mathrm{~cm}$. Other instrumentation used in the experiment is located out of view on the opposite side of SuperEBIT.

The main calibration spectrometer, the EBIT calorimeter spectrometer (ECS), is a solid-state device first developed at NASA's Goddard Space Flight Center in 1984 [5]. The present ECS consists of a $6 \times 6$ array of HgTe pixels cryogenically-cooled to $50 \mathrm{mK}$ using an adiabatic de-magnetization refrigerator in a liquid ${ }^{3} \mathrm{He} /{ }^{4} \mathrm{He}$ bath. The array consists of $625 \times 625 \mu \mathrm{m}^{2} \times 8$ $\mu \mathrm{m}$ thick pixels for mid-energy $0.1-10 \mathrm{keV}$ photons interspersed with $625 \times$ 


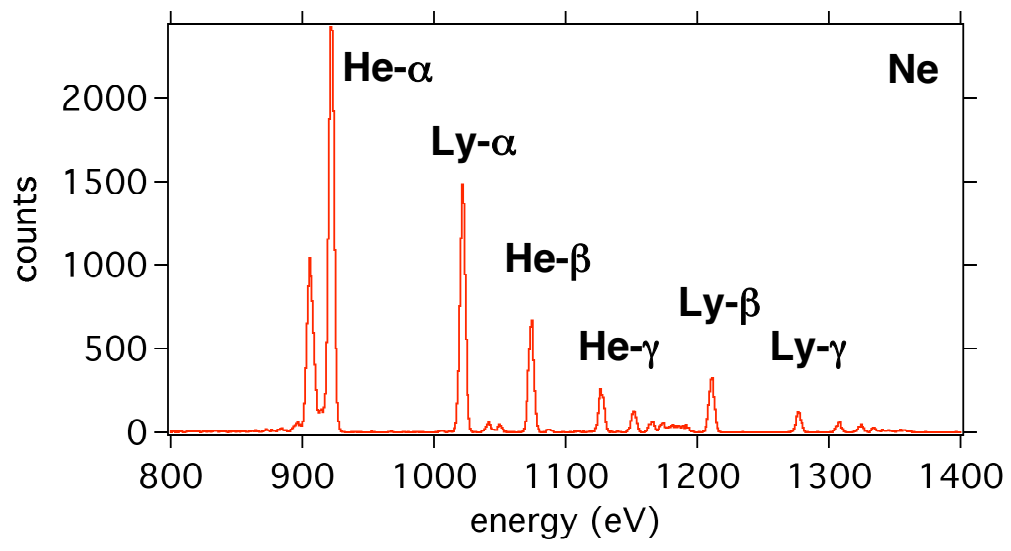

Fig. 2. Spectrum emitted from He-like and H-like Ne produced by SuperEBIT and measured with the ECS. Strongest transitions are labeled.

$500 \mu \mathrm{m}^{2} \times 100 \mu \mathrm{m}$ thick pixels for high energy $0.5-100 \mathrm{keV}$ photon detection. $\mathrm{HgTe}$ is chosen as the detector material because of high x-ray absorption and low heat capacity. X-ray absorption is $100 \%$ for photon energy below $4 \mathrm{keV}$. The energy resolution when operated at the $50 \mathrm{mK}$ temperature is $\Delta E \sim 5 \mathrm{eV}$ at $6 \mathrm{keV}$ and $\Delta E \sim 25 \mathrm{eV}$ at $60 \mathrm{keV}$ photon energy. Thermal isolation of the calorimeter is achieved with 4 thin foils of aluminized polyimide $\left(\mathrm{C}_{22} \mathrm{H}_{10} \mathrm{~N}_{2} \mathrm{O}_{5}\right)$ with a total thickness of $143.4 \mathrm{~nm} \mathrm{Al} / 218.2 \mathrm{~nm}$ polyimide. The absorption of this filter set has to be corrected in the calibration for low energy photons under study here. A typical K-shell spectrum for $\mathrm{Ne}$ is shown in Fig. 2 where the $\mathrm{x}$-ray photons are detected by

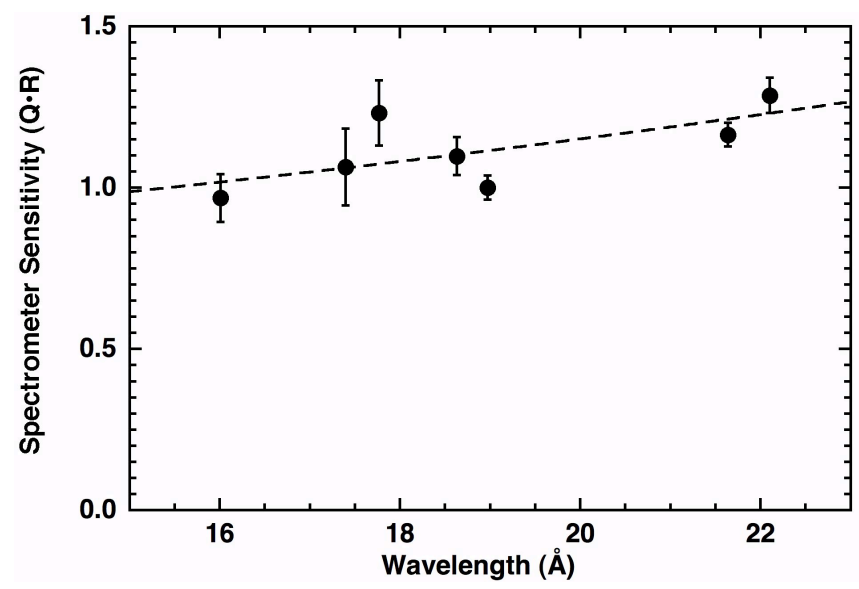

Fig. 3. Relative HRGS spectrometer sensitivity (detector efficiency and grating reflectivity) normalized against the oxygen Ly- $\alpha$ spectral line at $18.97 \AA$. Dashed line is second order polynomial fit to data points. 
the mid-energy pixels in the ECS array. High $n=7$ transitions can be clearly resolved in the He-like and $\mathrm{H}$-like series where $E / \Delta E$ is 200 at $1 \mathrm{keV}$ photon energy. For filter calibration, the filter was placed on a translation stage between the ECS and the x-ray source. Several runs of equal integration time were conducted with the filter in and out. The signal intensity and ionization balance of the x-ray source were monitored in all runs with the two grating spectrometers and the ECS data was corrected for small changes.

\section{Experimental Results}

A preliminary calibration of the relative sensitivity $Q(E) \cdot R(E)$ is determined for the HRGS by measuring the change in the sensitivity in the $15-23 \AA$ range normalized to the $\mathrm{O} \mathrm{H}$-like $1 s-2 p$ line at $18.97 \AA$. The integrated signal in the spectral lines was measured for both the ECS and HRGS instruments running and integrating x-ray signal simultaneously. The filter response of the ECS was corrected for in the number of detected photons to determine the photon fluence incident on the HRGS instrument. The photon energy in each spectral line was taken into account in the analysis of the HRGS results. Figure 3 shows the results of the relative spectrometer sensitivity for the HRGS. The dashed line is a quadratic fit to the data points.

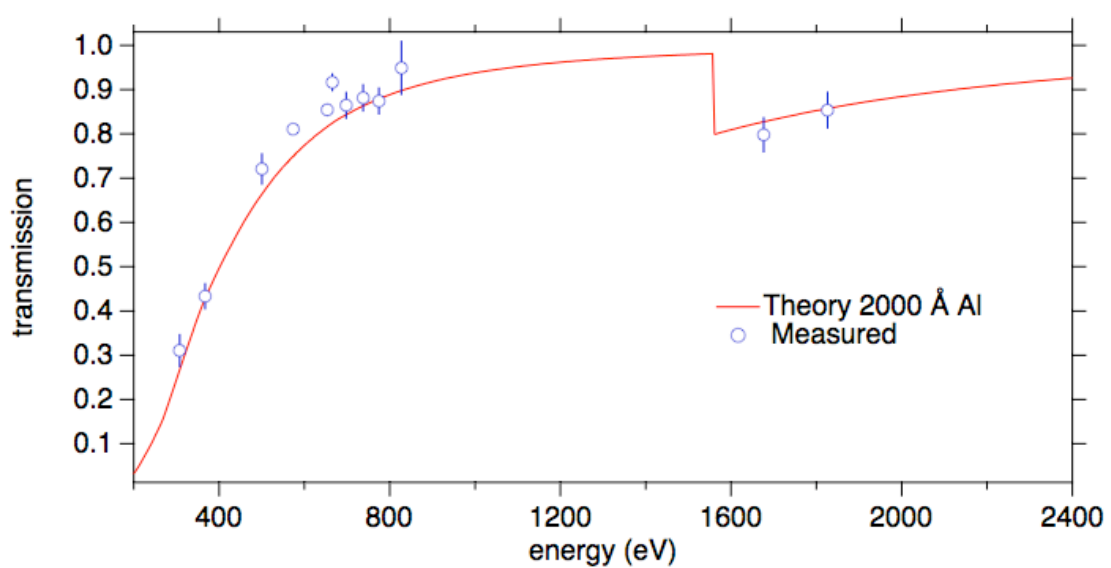

Fig. 4. Measured transmission of a $200 \mathrm{~nm} \mathrm{Al} \mathrm{filter} \mathrm{using} \mathrm{the} \mathrm{ECS.} \mathrm{Solid} \mathrm{line} \mathrm{is}$ theoretical data obtained from ref. 9.

Error bars are determined mainly by the photon statistics recorded in each line with the largest errors coming from the weaker, higher $n$ transitions. There is a general trend of sensitivity falling by approximately $25 \%$ with decreasing wavelength largely from the grating reflectivity response. The reported reflectivity for a smaller radius of curvature 2400 line/mm Hitachi grating in the same waveband shows a more rapid fall off in reflectivity [4]. 
Figure 4 shows the spectrometer filter transmission for the $200 \mathrm{~nm} \mathrm{Al}$ measured by the ECS as described in the previous section. Good agreement between the measured values and the predicted transmission curve [9] is shown for the energy range investigated here. The results presented in Figs. 3 and 4 indicate that the high resolution grating instrument response can be accurately calibrated on EBIT. The HRGS is found to have useable reflectivity to below $12 \AA$. More details of the calibration will be reported at a later date, including using the ECS for absolute efficiency calibration.

The authors would like to thank Joel Clementson and Miriam Frankel for their assistance in running SuperEBIT. This work performed under the auspices of the U.S. Department of Energy by Lawrence Livermore National Laboratory under Contract DE-AC52-07NA27344.

\section{References}

1. J. Dunn, E.W. Magee, R. Shepherd, H. Chen, S.B. Hansen, S.J. Moon, G.V. Brown, M.-F. Gu, and P. Beiersdorfer, and M.A. Purvis, "High resolution soft Xray spectroscopy of low Z K-shell emission from laser-produced plasmas", accepted for publication in Rev. Sci. Instrum. (2008).

2. H.R. Griem, "Plasma Spectroscopy", (McGraw-Hill, New York, 1964).

3. C. De Michelis and M. Mattioli, "Soft-x-ray spectroscopic diagnostics of laboratory plasmas", Nucl. Fus. 21(6), 677 (1981).

4. A. Saemann and K. Eidmann, "Absolute calibration of a flat field spectrometer in the wavelength range 10-70 ̊”. Rev. Sci. Instrum. 69, 1949 (1998).

5. F.S. Porter, B.R. Beck, P. Beiersdorfer, K.R. Boyce, G.V. Brown, H. Chen, J. Gygax, S.M. Kahn, R.L. Kelley, C.A. Kilbourne, E. Magee, and D.B. Thorn, "The XRS microcalorimeter spectrometer at the Livermore electron beam ion trap", Can. J. Phys. 86, 231 (2008).

6. G.V. Brown, P. Beiersdorfer, J. Emig, M. Frankel, M.F. Gu, R.F. Heeter, E. Magee, D.B. Thorn, K. Widmann, R.L. Kelley, C.A. Kilbourne, and F.S. Porter, "Rapid, Absolute Calibration of X-ray Filters Employed By Laser-Produced Plasma Diagnostics", accepted for publication in Rev. Sci. Instrum. (2008).

7. P. Beiersdorfer, "A "brief" history of spectroscopy on EBIT", Can. J. Phys. 86(1), 1 - 10 (2008); More references and work on EBIT can be found in this volume.

8. P. Beiersdorfer, E.W. Magee, E. Träbert, H. Chen, J.K. Lepson, M.-F. Gu, and M. Schmidt, "Flat-field grating spectrometer for high-resolution soft $\mathrm{x}$-ray and extreme ultraviolet measurements on an electron beam ion trap", Rev. Sci. Instrum. 75, 3723 (2004).

9. Theoretical filter transmission obtained from the Lawrence Berkeley National Laboratory website at http://www-cxro.lbl.gov/ 